\title{
EFECTIVIDAD DE ALGUNOS TRATAMIENTOS PRE-GERMINATIVOS para ocho especies leÑosas de la MixteCA Alta oAXaQueÑa CON CARACTERÍSTICAS RELEVANTES PARA LA RESTAURACIÓN
}

\author{
Gilberto Martínez-Pérez ${ }^{1}$ Alma Orozco-Segovia² y Carlos Martorelli,3 \\ 'Departamento de Ecología y Recursos Naturales, Facultad de Ciencias, Universidad Nacional Autónoma \\ de México, Circuito Exterior s/n, Ciudad Universitaria, México 04510, D.F., México. \\ ${ }^{2}$ Instituto de Ecología, Universidad Nacional Autónoma de México. \\ ${ }^{3}$ Autor para la correspondencia. Tel (55) 5622-4835, Fax (55) 5622-4828; \\ correo-e: martorel@ecologia.unam.mx
}

\begin{abstract}
Resumen: Para restaurar zonas fuertemente degradadas como la Mixteca Alta (Oaxaca, México) es importante usar especies nativas que faciliten la sucesión natural. Para ello necesitamos saber si sus semillas presentan latencia y cómo romperla. En ocho especies apropiadas para la restauración, evaluamos la efectividad de diferentes tratamientos pre-germinativos seleccionados con base en la literatura. Los resultados se analizaron con un nuevo método que resuelve algunos problemas estadísticos frecuentes en el análisis de estos experimentos. Acacia schaffneri, Ipomoea murucoides, Mimosa aculeaticarpa y Dodonaea viscosa presentan latencia física, ya que basta quebrantar la testa mediante abrasión o calentamiento para lograr una germinación rápida. A pesar de pertenecer a familias que sólo presentan latencia fisiológica, las semillas de Arctostaphylos pungens y Juniperus flaccida germinan sumergiéndolas en ácido, lo que quizá facilitó al embrión inmaduro la ruptura de la testa. Encontramos latencia fisiológica somera en Quercus deserticola y aparente ausencia de latencia en Quercus castanea.
\end{abstract}

Palabras clave: análisis estadístico, especies nativas, germinación, latencia, reforestación.

\begin{abstract}
When restoring highly degraded areas such as the Mixteca Alta (Oaxaca State, Mexico), it is important to use native species that promote natural succession. To do so, we need to know whether their seeds have dormancy and how to break it. We compared different pre-germination treatments of eight species relevant for restoration. The results were analyzed with a new method that solves some of the statistical problems that arise when examining these experiments. In Acacia schaffneri, Ipomoea murucoides, Mimosa aculeaticarpa and Dodonaea viscosa wearing off the seed coat by means of abrasion or heating promotes rapid germination, proving the presence of physical dormancy. Despite belonging to families that show physiological dormancy only, the seeds of Arctostaphylos pungens and Juniperus flaccida germinate after immersion in acid. This procedure may have weakened the seed coat, allowing the immature embryo to break it. We found weak physiological dormancy in Quercus deserticola, and no apparent dormancy in Quercus castanea.
\end{abstract}

Key words: dormancy, germination, native species, reforestation, statistical analysis.

$\mathbf{E}^{\mathrm{n}}$ n la disciplina de la recuperación de áreas degradadas existe la inquietud de restablecer especies nativas propias de la vegetación primaria o secundaria. Esto se debe a que dichas especies están adaptadas a las condiciones de la zona y nos permiten aprovechar procesos sucesionales naturales que conducen al restablecimiento de la vegetación original (Vázquez-Yanes et al., 1995; Cervantes et al., 2001). Esto es particularmente importante en zonas que han sufrido una intensa degradación ambiental que puede impedir el establecimiento inmediato de las especies propias de la vegetación madura.

A menudo, en los proyectos de restauración se introducen al campo individuos obtenidos a partir de semillas, las cuales frecuentemente pueden requerir de un tratamiento previo para germinar exitosamente (Baskin y Baskin, 1998). Sin embargo, en muchos casos se desconoce cuáles son los tratamientos apropiados para propagar las especies con valor para la restauración (González y Camacho, 1994), e incluso se carece de datos mínimos en torno a la ecología de dichas especies. Esto impide su utilización y manejo apropiado (Vázquez-Yanes y Cervantes, 1993; Cervantes, 1996; Vázquez-Yanes y Batis-Muñoz, 1996). 
Gilberto Martínez-Pérez, Alma Orozco-Segovia y Carlos Martorell

En México, una de las regiones más degradadas del país es la Mixteca Alta oaxaqueña, la cual ha estado sometida históricamente a una alta intensidad de disturbio, lo que ha ocasionado la pérdida de gran parte de la cobertura vegetal y del suelo que la sustentaba. Es posible reconocer varios estadios sucesionales en la vegetación remanente, así como extensas zonas de pastizal de origen antropogénico (CruzCisneros y Rzedowski, 1980; Martínez-Pérez, 2004). La falta de la información necesaria para germinar las semillas de varias especies nativas ha impedido su uso con fines de reforestación, reintroducción o restauración, a pesar de que existe el interés entre los pobladores e instituciones que tratan de incidir en la región. En la presente contribución se dan a conocer los avances logrados en estos aspectos, utilizando ocho especies de plantas leñosas que forman parte de las comunidades vegetales primarias y secundarias en la región.

Un primer paso para determinar las condiciones necesarias para que las semillas germinen es identificar si éstas presentan latencia, la cual se manifiesta cuando no ocurre la germinación de semillas viables a pesar de que éstas se encuentran en condiciones óptimas para ello. La latencia puede clasificarse según el mecanismo que impide la germinación (Baskin y Baskin, 1998, 2004). La latencia física es causada por una capa impermeable al agua en la testa de las semillas (Rolston, 1987; Baskin et al., 2000, 2004) y es conocida en 9 órdenes y 15 familias de angiospermas del mundo. Este tipo de latencia no ha sido documentado en las gimnospermas (Baskin et al., 2004). Por el contrario, la latencia fisiológica está presente en un mayor número de familias y es causada por un mecanismo que inhibe el metabolismo del embrión, impidiendo que éste se desarrolle y ocurra la germinación (Baskin y Baskin, 1998).

Debido a los severos procesos de deterioro de los suelos de la Mixteca Alta seleccionamos especies que fueran capaces de retener o mejorar el suelo, además de que incluyeran algunas de las características deseables en las especies utilizadas en la restauración de áreas perturbadas (Vázquez-Yanes, 1995). Con este propósito incorporamos al estudio especies que pueden formar nuevo suelo, es decir, incrementar su volumen. Éste puede ser el caso de especies que presentan una buena producción de hojarasca como Arctostaphylos pungens y Dodonaea viscosa (obs. pers.). Otras especies pueden enriquecer el suelo, aportándole diferentes nutrientes, como sucedería en especies que presentan nódulos fijadores de nitrógeno. Entre éstas probablemente se encuentren las leguminosas Acacia schaffneri y Mimosa aculeaticarpa (Virginia y Jarrel, 1983; Pugnaire et al., 1996; Cervantes et al., 1998). Estas especies se encuentran de manera natural en sitios perturbados, por lo que, al igual que otras especies de la vegetación secundaria, probablemente sean capaces de resistir condiciones limitantes tales como baja fertilidad, estrés hídrico, suelos compactados, $\mathrm{pH}$ extremo o salinidad elevada, entre otros fac- tores (Vázquez-Yanes, 1995). Otras especies arbóreas típicas de vegetación secundaria son Ipomoea murucoides y Juniperus flaccida. Esta última especie, junto con Arctostaphylos pungens, produce propágulos comestibles, por lo que puede atraer a la fauna dispersora de semillas, favoreciendo el restablecimiento de elementos de la flora y fauna nativas. Finalmente, elegimos a Quercus castanea y Quercus deserticola debido a que tienen un elevado valor para la conservación, siendo especies típicas de la vegetación madura (cuadro 1). Es posible que varias de las especies seleccionadas puedan facilitar la introducción de otras, es decir, que sean capaces de mejorar las condiciones microambientales (formación o enriquecimiento del suelo, reducción de la radiación solar, etc.) permitiendo el establecimiento de otras plantas.

Para determinar cuál es un mecanismo apropiado para romper la latencia en cada especie se realizó una revisión de los métodos publicados en la literatura para cada una. En el caso de que no hubiera antecedentes, se buscó información de otros organismos del mismo género. De las ocho especies seleccionadas, se ha reportado que cuatro tienen latencia en sus semillas y que una (Quercus deserticola) no la presenta (Zavala-Chávez, 2004; cuadro 1). Para congéneres de las tres especies restantes también se ha reportado latencia (Djavanshir y Fechner, 1975; Pardos y Lázaro, 1983; Rey-Cruz, 1985; Murguía-Sánchez, 1986; Young et al., 1988; Young y Young, 1992; González y Camacho, 1994; Cervantes et al., 1998; Cervantes et al., 2001). En algunas especies se reportan varios tratamientos alternativos. En estos casos sólo se evaluaron aquellos que fueran más sencillos de aplicar, ya que es posible que campesinos o manejadores puedan estar interesados en emplearlos, pero no siempre cuentan con equipo sofisticado. También se dio preferencia a aquellos tratamientos que han probado su efectividad en México sobre aquellos que se basan en germoplasma de lugares más distantes. Por ejemplo, se puede lograr que Dodonaea viscosa germine mediante escarificación mecánica e inmersión en agua caliente (Camacho y Bustillo, 1988; González y Camacho, 1994; Burrows, 1995; Baskin et al., 2004; Phartyal et al., 2005). La eficiencia de estos dos métodos varía según la región geográfica; sin embargo, en México la inmersión en agua ha probado ser más eficiente, además de ser un método más sencillo que la escarificación manual de las semillas. Por ello, se prefirió examinar la efectividad de la escarificación por temperatura en vez de la mecánica.

\section{Materiales y métodos}

Zona de estudio. Este trabajo se realizó en el Municipio de Concepción Buenavista, Oaxaca, México, cuyas características geológicas hacen que el suelo sea muy deleznable. En la mayor parte de la zona el suelo es somero y pedregoso, con profundidad menor que $30 \mathrm{~cm}$. No se observan hori- 


\section{TRATAMIENTOS PRE-GERMINATIVOS PARA ESPECIES RELEVANTES PARA LA RESTAURACIÓN}

Cuadro 1. Características relevantes de las especies estudiadas. Se destacan para cada una algunos atributos deseables para la restauración tales como el mejoramiento de suelos o de condiciones microclimáticas (facilitación). Se señalan los métodos reportados para romper la latencia de estas especies y el hábitat en que se encuentran. 1: restitución de biota originaria; 2: restitución de procesos ecosistémicos (fijación de nitrógeno o formación de suelo); $R$ : retención del suelo; $P$ : formación de suelo; $E$ : enriquecimiento del suelo; $F$ : facilitación; $O$ : vegetación primaria.

\begin{tabular}{|c|c|c|c|c|c|c|c|c|c|}
\hline \multirow[t]{2}{*}{ Especie } & \multirow[t]{2}{*}{ Objetivo } & \multicolumn{5}{|c|}{ Características } & \multirow{2}{*}{$\begin{array}{c}\text { Ruptura } \\
\text { de latencia }\end{array}$} & \multirow[t]{2}{*}{ Referencias } & \multirow[t]{2}{*}{ Hábitat } \\
\hline & & $\boldsymbol{R}$ & $\boldsymbol{P}$ & $E$ & $\boldsymbol{F}$ & $O$ & & & \\
\hline $\begin{array}{l}\text { Acacia } \\
\text { schaffneri }\end{array}$ & 2 & $\checkmark$ & & $\checkmark$ & $\checkmark$ & & $\begin{array}{l}\text { Escarificación } \\
\text { mecánica y ácida }\end{array}$ & $\begin{array}{l}\text { Everitt (1983), Vora (1989), Young y } \\
\text { Young (1992), Martorell (1995), } \\
\text { Cervantes et al. (2001) }\end{array}$ & $\begin{array}{l}\text { Muy perturbado, } \\
\text { suelos exiguos }\end{array}$ \\
\hline $\begin{array}{l}\text { Mimosa } \\
\text { aculeaticarpa }\end{array}$ & 2 & $\checkmark$ & & $\checkmark$ & $\checkmark$ & & $\begin{array}{l}\text { Escarificación } \\
\text { mecánica }\end{array}$ & $\begin{array}{l}\text { Cervantes et al. (1998), González y } \\
\text { Camacho, (1994), Cervantes et al. (2001) }\end{array}$ & $\begin{array}{l}\text { Muy perturbado, } \\
\text { suelos exiguos }\end{array}$ \\
\hline $\begin{array}{l}\text { Ipomoea } \\
\text { murucoides }\end{array}$ & 2 & $\checkmark$ & & & & & $\begin{array}{l}\text { Escarificación } \\
\text { mecánica }\end{array}$ & $\begin{array}{l}\text { Rey-Cruz (1985), Murguía-Sánchez } \\
\text { (1986), SEMARNAT (2000) }\end{array}$ & Zonas perturbadas \\
\hline $\begin{array}{l}\text { Juniperus } \\
\text { flaccida }\end{array}$ & 1 & $d$ & & & $\checkmark$ & $\checkmark$ & $\begin{array}{l}\text { Escarificación } \\
\text { ácida }\end{array}$ & $\begin{array}{l}\text { Young y Young (1992), Young et al. } \\
\text { (1988), Pardos y Lázaro (1983), } \\
\text { Djavanshir y Fechner (1975) }\end{array}$ & Bosques abiertos \\
\hline $\begin{array}{l}\text { Quercus } \\
\text { deserticola }\end{array}$ & 1 & $\checkmark$ & $\checkmark$ & & $\checkmark$ & $\checkmark$ & Ninguno & Zavala-Chávez (2004) & Bosques abiertos \\
\hline $\begin{array}{l}\text { Quercus } \\
\text { castanea }\end{array}$ & 1 & $\checkmark$ & $\checkmark$ & & $\checkmark$ & $\checkmark$ & $\begin{array}{l}\text { Escarificación } \\
\text { mecánica }\end{array}$ & Gosling (1989), Zavala-Chávez (2004) & Bosques de encino \\
\hline $\begin{array}{l}\text { Dodonaea } \\
\text { viscosa }\end{array}$ & 2 & $\checkmark$ & $\checkmark$ & & & & $\begin{array}{l}\text { Escarificación } \\
\text { mecánica y fuego }\end{array}$ & $\begin{array}{l}\text { Qadir y Lodhi (1971), Hodkinson (1991), } \\
\text { Hodkinson y Oxley (1990), González y } \\
\text { Camacho (1994), Burrows (1995), Rosa y } \\
\text { Ferreira (2001) }\end{array}$ & $\begin{array}{l}\text { Muy perturbado, } \\
\text { suelos exiguos } \\
\text { o nulos }\end{array}$ \\
\hline $\begin{array}{l}\text { Arctostaphylos } \\
\text { pungens }\end{array}$ & 2 & $\checkmark$ & $\checkmark$ & & & & $\begin{array}{l}\text { Estratificación, } \\
\text { escarificación } \\
\text { ácida, fuego }\end{array}$ & $\begin{array}{l}\text { Carlson y Sharp (1975), Young y } \\
\text { Young (1992) }\end{array}$ & $\begin{array}{l}\text { Bosques de encino } \\
\text { perturbados }\end{array}$ \\
\hline
\end{tabular}

zontes edáficos. Los eventos de lluvia son torrenciales y ocurren en lapsos de tiempo muy breves, lo que contribuye grandemente a la pérdida y lavado del suelo. El tipo de clima que prevalece es semiárido (Cruz-Cisneros y Rzedowski, 1980). La vegetación natural muestra una intensa perturbación y se observan varios estadios sucesionales. Son frecuentes los sitios donde se pasa paulatinamente de una comunidad a otra y de terrenos con vegetación a lugares con roca madre al descubierto. El paisaje que se observa en la actualidad es resultado del intenso disturbio antropogénico crónico al que fue y sigue estando sometida la vegetación de la región, debido a lo cual podemos encontrar sitios con diferentes grados de conservación (MartínezPérez, 2004).

El material biológico se recolectó en los meses de agosto de 2003 a enero de 2004. Para cada especie se colectaron alrededor de 1,000 semillas de al menos 15 individuos, procurando tomarlas directamente del árbol para evitar la contaminación por agentes patógenos vinculados al suelo
(Zavala-Chávez y García, 1996). Posteriormente las semillas fueron secadas a temperatura ambiente, sin exponerlas directamente a los rayos solares, y se almacenaron a temperatura ambiente en bolsas de papel de estraza. Las semillas de las ocho especies seleccionadas fueron separadas por medio de flotación para descartar a las semillas vanas o no viables (Zavala-Chávez y García, 1996).

Descripción de las especies. Dodonaea viscosa Jacq. (Sapindaceae): jarilla. Es un árbol pequeño o un arbusto de 1 a $5 \mathrm{~m}$ de altura, perennifolio, muy variable en su morfología, fenología y ecología (Baskin et al., 2004). En México crece en comunidades secundarias, sobre todo en encinares perturbados por fuego y erosionados (NiembroRocas, 1986; Camacho y Bustillo, 1988; Marroquín, 2001). Se reporta que sus semillas presentan latencia física, la cual puede romperse mediante remojo en agua por $24 \mathrm{~h}$ a temperatura ambiente (Qadir y Lodhi, 1971), fuego (Hodgkinson, 1991), o por remojo en agua caliente por $5 \mathrm{~s}$ 
Gilberto Martínez-Pérez, Alma Orozco-Segovia y Carlos Martorell

ó 4 min (Hodgkinson y Oxley, 1990; González y Camacho, 1994), escarificación mecánica de la testa (Burrows, 1995) e inmersión en ácido sulfúrico (Rosa y Ferreira, 2001). Sólo Hussain et al. (1991) reportan que esta especie no requiere ningún tratamiento para germinar.

Ipomoea murucoides Roem. et Schult. (Convolvulaceae): cazahuate. Es un árbol de selvas bajas y pastizales antropogénicos que crece con facilidad en suelos muy perturbados (SEMARNAT, 2000). Sus semillas presentan latencia física que puede romperse mediante escarificación mecánica de la testa (Rey-Cruz, 1985; Murguía-Sánchez, 1986; SEMARNAT, 2000).

Acacia schaffneri (S.Watson) F.J.Herm. (Mimosaceae): huizache. Árboles o arbustos de hasta $4 \mathrm{~m}$ de alto, que habitan en los bosques de Quercus, matorral xerófilo y pastizal. Abundante en lugares perturbados o erosionados (RicoArce y Rodríguez, 1998). Las especies de Acacia tienen una testa dura e impermeable, por lo que presentan latencia física. Ésta puede romperse por inmersión en agua caliente, calor seco, o calor con microondas (Young y Young, 1992; Martorell, 1995; Cervantes et al., 2001). Más frecuentes son los reportes de que requiere escarificación ácida para romper su latencia (Everitt, 1983; Vora, 1989), o escarificación mecánica (Flores y Jurado, 1998; Jurado et al., 2000). Cada uno de los tratamientos produce altos porcentajes de germinación, aunque González-Castañeda et al. (2004) reporta un porcentaje más alto con escarificación ácida.

Mimosa aculeaticarpa Ortega (Fabaceae): garabato. Arbusto de 0.5 a $2 \mathrm{~m}$ de altura, asociado a vegetación secundaria de bosques de encino (SEMARNAT, 2000). Para Mimosa aculeaticarpa no existen reportes o trabajos previos sobre su germinación, pero para otras especies del mismo género se reporta la presencia de latencia física que se rompe mediante escarificación mecánica (Cervantes et al., 1998; González y Camacho, 1994; Cervantes et al., 2001; Orozco-Almanza et al., 2003; Camargo-Ricalde et al., 2004) o escarificación ácida (González-Castañeda et al., 2004; Jurado et al., 2000).

Arctostaphylos pungens Kunth (Ericaceae): manzanita. Son arbustos y ocasionalmente árboles de 1 a $3.5 \mathrm{~m}$ de altura, perennifolios (Young y Young, 1992). Forman parte importante del chaparral y de los bosques de Quercus. Para el género existen reportes de la presencia de una testa muy dura y probablemente latencia fisiológica (Young y Young, 1992). Los tratamientos empleados para romperla consisten en debilitar la barrera mecánica que representa la cubierta seminal con inmersión en ácido sulfúrico, o bien tratamientos que rompen la latencia fisiológica, como estratificación con calor, frío y 24 h de inmersión en agua
(Keeley, 1987; Odion y Tyler, 2002). Los tiempos de exposición en ácido van de 2 a 6 h (Carlson y Sharp, 1975; Young y Young, 1992).

Juniperus flaccida Schltdl. (Cupressaceae): enebro. Son árboles de hasta de $12 \mathrm{~m}$ de altura (Medina-Lemus y Dávila-Aranda, 1997). Se localizan en asociaciones vegetales de encino con suelos generalmente pobres, rocosos y arenosos. Las semillas del género Juniperus presentan latencia fisiológica (Young y Young, 1992) y en estudios previos se reporta que se ha roto con enfriamiento por 14 semanas (Young et al., 1988) o por enfriamiento en una solución de giberelinas a $5^{\circ} \mathrm{C}$, con oxígeno a saturación (Young y Young, 1992), o estratificación con calor seco (Pardos y Lázaro, 1983). La inmersión en ácido sulfúrico por 35 a 120 min seguida de la remoción de los restos carbonizados, también permite la emergencia del embrión inmaduro (Djavanshir y Fechner, 1976).

Quercus deserticola Trel. (Fagaceae): chaparro. Es un arbolillo de 6-8 m, endémico de México, que crece en asociaciones vegetales de pastizal o bosque abierto de encinos (Vázquez-Villagrán, 2000). Por ser una especie que pertenece al subgénero Lepidobalanus no presenta latencia, o en caso de que ésta se presente, generalmente es muy corta y sólo se recomienda remojo por 24 h para homogenizar el proceso de germinación. Las bellotas de las especies del subgénero suelen mantener su viabilidad con contenidos de humedad de 40 a 45\% (Zavala-Chávez, 2004).

Quercus castanea Née (Fagaceae): encino de tinte. Árbol de 5 a $20 \mathrm{~m}$ de alto (Vázquez-Villagrán, 2000), que se encuentra en asociaciones de encino y encino-enebro, en suelos profundos y bien drenados (SEMARNAT, 2000). Esta especie forma parte del subgénero Erythrobalanus y presenta latencia fisiológica que comúnmente se rompe a través de enfriamiento o escarificación mecánica (Young y Young, 1992). Aunque pueden permanecer viables con sólo un $25 \%$ de humedad, se ha demostrado que las bellotas cuyo contenido de humedad es alto germinan más fácilmente que las de humedad menor, por lo que es recomendable iniciar la germinación poco después de la colecta de las semillas (Gosling, 1989; Zavala-Chávez, 2004).

Ruptura de latencia. Durante marzo de 2004, se evaluó en el laboratorio la presencia de latencia en las semillas de las especies seleccionadas y las formas de romperla, así como el porcentaje de germinación para cada una de ellas. Las semillas de las siete especies para las que había evidencia bibliográfica de latencia fueron sometidas a diversos tratamientos. Éstos fueron seleccionados de acuerdo con la efectividad reportada en trabajos previos.

A todas las semillas de las especies bajo estudio se les 


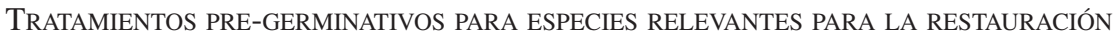

aplicó remojo en agua por 24 h, y desinfección durante 5 min en hipoclorito de sodio, antes de sembrarlas en agar al $1 \%$. Ésta es la única manipulación que se le hizo a las semillas del tratamiento control. De cada especie se colocaron cinco repeticiones por tratamiento con 15 semillas cada una, y en el caso de $Q$. deserticola se colocaron 10 semillas por tratamiento debido a que no cabían más en una caja Petri.

Las semillas de las ocho especies fueron colocadas en cámaras de ambiente controlado (LAB LINE Instruments, Inc.; Melrose Park, Illinois, E.U.A.) a una temperatura constante de $18^{\circ} \mathrm{C}$, que es la temperatura promedio en la zona de estudio, con un fotoperiodo de 12/12. Las cámaras estuvieron provistas de luz fluorescente de día (Silvana, F20T12/CW de 20 W) y lámparas incandescentes tubulares (General Electric B9 de $25 \mathrm{~W}$ ) para simular la calidad espectral de la luz solar; el flujo fotónico en las cámaras fue de $33.21 \mu \mathrm{mol} \mathrm{m} \mathrm{m}^{-2} \mathrm{~s}^{-1}$. La emergencia de la radícula se empleó como indicador de la germinación. Las semillas se revisaron cada tres días, excepto en Acacia schaffneri y Dodonaea viscosa, las cuales se revisaron diariamente. El seguimiento continuó hasta que la mayoría de las semillas había germinado, o bien hasta que se cumplieron 90 días de iniciado el experimento.

A las semillas de Acacia schaffneri, Ipomoea murucoides, Quercus castanea y Mimosa aculeaticarpa, para las cuales se reporta latencia física, con una lija del número 2 se aplicó abrasión mecánica hasta que la testa estaba claramente perforada (el embrión o el endospermo eran visibles). En el caso de D. viscosa las semillas fueron sumergidas en agua caliente a $70^{\circ} \mathrm{C}$ por 4 min (González y Camacho, 1994). A las semillas de Arctostaphylos pungens y Juniperus flaccida se les aplicó el tratamiento de inmersión en ácido sulfúrico con una concentración de $98 \%$ por periodos de $0,2,3,4,5,6,8,10$ y $12 \mathrm{~h}$, seguidos de un lavado durante 50 min bajo agua corriente para eliminar los residuos carbonizados. A Quercus deserticola, la única especie para la que no hay reportes de latencia, no se le aplicó ningún tratamiento además del remojo y desinfección.

Análisis estadístico. Existe una gran diversidad de métodos estadísticos para analizar la germinación que varían dependiendo del interés particular de cada investigador y la información que proporcionan. Se han propuesto varios índices para medir el proceso de germinación como el coeficiente de germinación, el índice de Abbot, el índice de germinación, la velocidad y tasa de emergencia, o la transformación Probit, entre otras (Camacho y Morales, 1992; González-Zertuche y Orozco-Segovia, 1996; Baskin y Baskin, 1998). Algunos de ellos implican una violación a los supuestos de las pruebas estadísticas empleadas, ya que no hay independencia en los datos. Para facilitar la obtención de diferentes indicadores de la germinación tales como el porcentaje total o la tasa de germinación, que anteriormente se obtenían a partir de varios análisis, nosotros proponemos el uso de un análisis de regresión logística basado en datos independientes que permite: (1) describir el curso de la germinación mediante el ajuste de una curva, (2) usar la secuencia completa de datos, (3) simplificar el análisis, y (4) obtener varios parámetros que describen la germinación a través un análisis único. La regresión se efectuó en el programa estadístico GLIM 4.0. Para las especies en las cuales se ensayaron más de dos tratamientos, éstos se compararon agregando todos los datos de diferentes niveles en un tratamiento único y evaluando si esto acarreaba un incremento significativo en la devianza residual (Crawley, 1993).

La regresión logística reconoce que los datos no son normales, sino que el número de semillas que germina es una fracción del total y por lo tanto sigue una distribución binomial. En ocasiones anteriores se ha empleado el número de semillas sembradas al inicio del experimento como el total para calcular la probabilidad de germinación a lo largo del tiempo. Esta práctica genera una falta de independencia en los datos, ya que el número de semillas que germinan en un momento dado debe ser menor o igual al número de semillas que no lo ha hecho con anterioridad. Este problema se resuelve calculando la probabilidad de germinación en el tiempo $t$ no con respecto al número inicial de semillas sino respecto a aquellas que no han germinado en el tiempo $t-1$, de modo que el resultado de la división puede tomar cualquier valor entre cero y uno. Esto es, mediante la regresión se ajusta la probabilidad de que una semilla germine en el tiempo $t$ dado que no ha germinado con anterioridad, lo cual es una probabilidad condicional. Empleando el teorema de Bayes, esta probabilidad puede transformarse posteriormente en la probabilidad de que una semilla cualquiera germine en el tiempo $t$, que es el dato que normalmente se emplea en los estudios de germinación (para los detalles véase el apéndice 1).

\section{Resultados}

El método estadístico propuesto en este estudio permitió detectar diferencias entre los tratamientos a los que se expusieron las especies (cuadro 2). Los valores de $R^{2}$ de las regresiones fueron superiores a 0.70 en todos los casos, excepto para $Q$. deserticola y $M$. aculeaticarpa, en las que $R^{2}$ fue menor, pero significativa, por lo que el modelo ajustado describe cercanamente los datos observados (figura 1). La inmersión en agua caliente promovió la germinación de D. viscosa (figura 1c). El resultado del tratamiento de abrasión en las especies con reportes de latencia física produjo una germinación superior a $80 \%$, la cual se inició durante la primera semana (figura 1a, d y f). La excepción fue $Q$. castanea, cuyas semillas presentaron una germinación total de $49 \%$, mientras que el control alcanzó un 


\section{Gilberto Martínez-Pérez, Alma Orozco-Segovia y Carlos Martorell}

Cuadro 2. Análisis de la devianza de las regresiones logísticas para la germinación de las ocho especies estudiadas. $t=$ tiempo transcurrido desde el inicio del experimento, $v=$ tratamiento. Sólo se muestran los términos significativos.

\begin{tabular}{|c|c|c|c|c|c|c|c|}
\hline \multirow{2}{*}{$\begin{array}{l}\text { Acacia schaffneri } \\
\text { Fuente }\end{array}$} & \multicolumn{3}{|c|}{$R^{2}=0.7115$} & \multicolumn{2}{|c|}{ Mimosa aculeaticarpa } & \multicolumn{2}{|c|}{$R^{2}=0.5975$} \\
\hline & $\chi^{2}$ & G.L. & $P$ & Fuente & $\chi^{2}$ & G.L. & $P$ \\
\hline$t$ & 67.39 & 1 & $<0.0001$ & $t$ & 85.83 & 1 & $<0.0001$ \\
\hline$t^{2}$ & 72.98 & 1 & $<0.0001$ & $t^{2}$ & 78.86 & 1 & $<0.0001$ \\
\hline$v$ & 154.40 & 1 & $<0.0001$ & $v$ & 22.39 & 1 & $<0.0001$ \\
\hline$v \times t$ & 14.16 & 1 & 0.0002 & $v \times t$ & 5.07 & 1 & 0.0244 \\
\hline \multicolumn{2}{|l|}{ Ipomoea murucoides } & \multicolumn{2}{|c|}{$R^{2}=0.8085$} & \multicolumn{2}{|c|}{ Quercus castanea } & \multicolumn{2}{|c|}{$R^{2}=0.7033$} \\
\hline Fuente & $\chi^{2}$ & G.L. & $P$ & Fuente & $\chi^{2}$ & G.L. & $P$ \\
\hline$t$ & 36.58 & 1 & $<0.0001$ & $t$ & 75.74 & 1 & $<0.0001$ \\
\hline$t^{2}$ & 25.33 & 1 & $<0.0001$ & $t^{2}$ & 66.88 & 1 & $<0.0001$ \\
\hline$v$ & 179.00 & 1 & $<0.0001$ & $v$ & 8.54 & 1 & 0.0035 \\
\hline \multicolumn{2}{|l|}{ Quercus deserticola } & \multicolumn{2}{|c|}{$R^{2}=0.622$} & \multicolumn{2}{|c|}{ Juniperus flaccida } & \multicolumn{2}{|c|}{$R^{2}=0.7033$} \\
\hline Fuente & $\chi^{2}$ & G.L. & $P$ & Fuente & $\chi^{2}$ & G.L. & $P$ \\
\hline$t$ & 43.10 & 1 & $<0.0001$ & $t$ & 71.06 & 1 & $<0.0001$ \\
\hline$t^{2}$ & 46.50 & 1 & $<0.0001$ & $t^{2}$ & 75.56 & 1 & $<0.0001$ \\
\hline$v$ & 54.70 & 8 & $<0.0001$ & $v \times t$ & 17.13 & 8 & 0.0288 \\
\hline \multicolumn{2}{|l|}{ Arctostaphylos pungens } & \multicolumn{2}{|c|}{$R^{2}=0.7115$} & \multicolumn{2}{|c|}{ Dodonaea viscosa } & \multicolumn{2}{|c|}{$R^{2}=0.821$} \\
\hline Fuente & $\chi^{2}$ & G.L. & $P$ & Fuente & $\chi^{2}$ & G.L. & $P$ \\
\hline$t$ & 67.39 & 1 & $<0.0001$ & $t$ & 67.39 & 1 & $<0.0001$ \\
\hline$t^{2}$ & 72.98 & 1 & $<0.0001$ & $t^{2}$ & 72.98 & 1 & $<0.0001$ \\
\hline$v$ & 154.40 & 1 & $<0.0001$ & $v$ & 15.40 & 1 & $<0.0001$ \\
\hline$v \times t$ & 14.16 & 1 & $<0.0001$ & $v \times t$ & 14.16 & 1 & $<0.0035$ \\
\hline
\end{tabular}

Cuadro 3. Tratamiento más efectivo para romper la latencia en cada especie. Las semillas se remojaron durante $24 \mathrm{~h}$ antes de la siembra. $t_{50}$ es el tiempo necesario para que germinaran $50 \%$ de las semillas en los tratamientos con mayor germinación.

\begin{tabular}{lccc}
\hline Especie & Tratamiento efectivo & Germinación total (\%) & t50 (días) \\
\hline Acacia schaffneri & Abrasión & 87 & 9 \\
Ipomoea murucoides & Abrasión & 97 & 18 \\
Mimosa aculeaticarpa & Abrasión & 96 & 21 \\
Quercus castanea & Ninguno & 67 & 30 \\
Quercus deserticola & Ninguno & 53 & 40 \\
Arctostaphylos pungens & Inmersión en ácido sulfúrico por & 63 & 18 \\
Juniperus flaccida & 6 h y 50 min de lavado & 79 & $50\left[38^{1}\right]$ \\
Inmersión en ácido sulfúrico por & 5 h y 50 min de lavado & & 8 \\
Dodonaea viscosa & Inmersión en agua a $70^{\circ} \mathrm{C}$ & & \\
& por 4 min & 98 &
\end{tabular}

'El dato entre corchetes corresponde al tratamiento de 6 h de escarificación, que fue el que germinó más rápidamente. 
total de $69 \%$. En esta especie el tratamiento también retrasó el inicio de la germinación (figura $1 \mathrm{~g}$, cuadro 3). Algunas de las semillas de estas especies presentaban daños no visibles a simple vista causados por depredadores que deterioraron la viabilidad de la semilla, aunque no fueron detectadas con la prueba de flotabilidad. Días después de la siembra, el incremento en volumen de la semilla causado por la imbibición evidenció en la testa la presencia de perforaciones pequeñas causadas por insectos.

Los tratamientos pre-germinativos más efectivos para las especies con reportes de latencia fisiológica, A. pungens y $J$. flaccida, fueron la inmersión en ácido sulfúrico concentrado por un tiempo de 6 y $5 \mathrm{~h}$ respectivamente (figura $1 \mathrm{~b}$ y e). En el caso de la primera especie, no encontramos diferencias significativas entre los tratamientos de 6, 8 y 12 $\mathrm{h}$ de inmersión. La germinación dio inicio en las primeras dos a tres semanas. A $Q$. deserticola no se le aplicó ningún tratamiento, con lo que se obtuvo una germinación de 53\% (figura $1 \mathrm{~h}$, cuadro 3 ).

\section{Discusión}

El método estadístico que se empleó en este trabajo puede constituirse como un método útil y de fácil aplicación en estudios futuros. Como puede apreciarse en la figura 1, los modelos ajustados describen bastante bien las curvas de germinación observadas. En algunos casos hay pequeñas diferencias que pueden ser importantes en ciertos estudios. Por ejemplo, la curva ajustada para Arctostaphylos pungens predice que la germinación daría inicio un poco antes de lo observado (figura 1b). Estas discrepancias menores se observan en cualquier regresión, y en general pueden

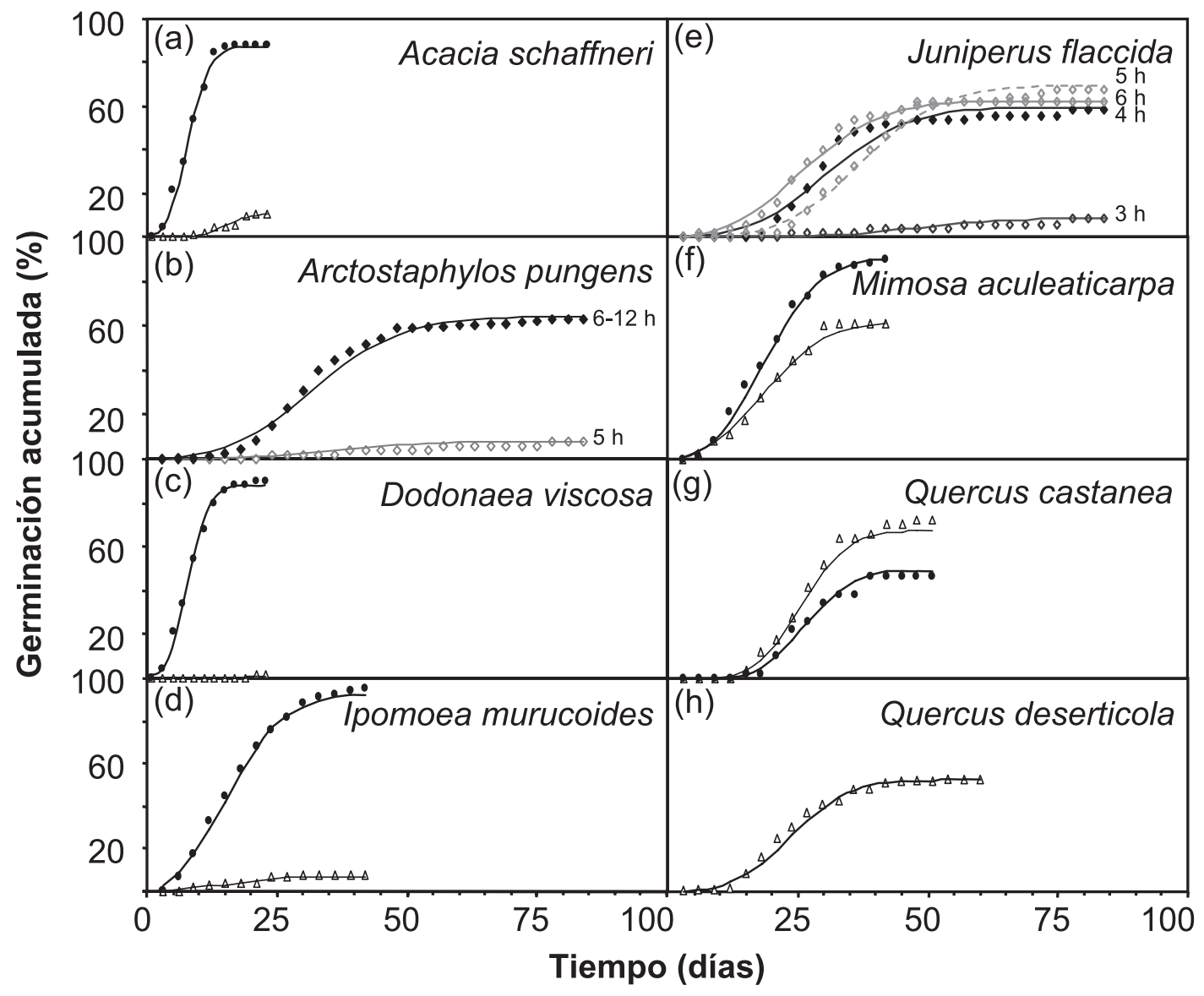

Figura 1. Curvas de germinación para las ocho especies estudiadas bajo diferentes tratamientos. Los triángulos blancos representan los controles y los círculos rellenos corresponden a los tratamientos de escarificación mecánica, la cual se realizó por abrasión excepto en Dodonaea viscosa, a la cual se le aplicó agua a $70^{\circ} \mathrm{C}$ por cuatro minutos. Los tratamientos de inmersión en ácido sulfúrico se indican con rombos, y el tiempo de exposición al mismo se indica al lado derecho de cada gráfica. Para lograr una mayor claridad, no se muestran los tratamientos de inmersión en ácido en los cuales no se registró ninguna germinación. 
corregirse ajustando una curva diferente. En este trabajo se ajustaron parábolas a los logits de las proporciones, pero el empleo de otras curvas pudiera resultar en un ajuste más preciso, si es que se considera necesario. Esta versatilidad del método puede tener otras aplicaciones. Por ejemplo, algunas especies germinan en pulsos; un porcentaje muy alto de semillas no latentes germina rápidamente, con un segundo evento menos masivo de germinación asociado a semillas con latencia que se presenta días más tarde (Baskin y Baskin, 2004). El empleo de polinomios de cuarto grado en la regresión logística puede modelar apropiadamente este tipo de germinación bimodal.

Un problema del método empleado es que requiere que los periodos entre observaciones sean regulares; de lo contrario, la interpretación del resultado se complica, y las curvas pueden reflejar tanto el comportamiento de la especie como los intervalos (establecidos arbitrariamente) entre observaciones. Otro posible problema es que los datos no sigan una distribución binomial. La violación de este supuesto generalmente produce sobredispersión (Crawley, 1993). En nuestro estudio no se encontró este problema, lo que sugiere que los residuos se comportan de la manera esperada. Desgraciadamente, ya que el parámetro $p$ de la distribución binomial cambia en el tiempo, no es posible aplicar una prueba a todos los residuos para examinar la validez del supuesto de binomialidad.

Se comprobó la existencia de latencia en siete de las ocho especies, de las cuales cuatro presentaron latencia física, dos mostraron latencia fisiológica, y una presentó latencia fisiológica en una fracción de la población de semillas utilizada.

Acacia schaffneri, Dodonaea viscosa, Mimosa aculeaticarpa e Ipomoea murucoides tienen testas impermeables (Everitt, 1983; Vora, 1989; Baskin et al., 2004; Cervantes et al., 1998). Al recibir el tratamiento de escarificación mecánica, estas especies iniciaron su germinación en menos de dos semanas. Por ello, siguiendo los criterios de Baskin y Baskin (2004), pueden clasificarse como especies con latencia física. De acuerdo con la literatura, la germinación en estas cuatro especies o sus congéneres ocurre en un $60-90 \%$ de las semillas tratadas (Cervantes et al., 2001; González y Camacho, 1994). Tres de estas especies superaron estos porcentajes y la otra tuvo altos valores dentro de este intervalo (87\%), por lo que consideramos que la aplicación de escarificación mecánica en las semillas de las poblaciones de la Mixteca Alta fue exitosa.

En general, nuestras observaciones en el laboratorio sugieren que el tamaño de la apertura de la testa puede influir en la velocidad de germinación. Este fenómeno ha sido observado en otras especies (Rolston, 1987). Otros estudios, sin embargo, señalan que una perforación pequeña debería ser suficiente para iniciar el proceso (Baskin y Baskin, 1998). Aunque nuestras observaciones no son formales, recomendamos realizar una apertura grande y de tamaño uniforme para acelerar el proceso de germinación. Sin embargo, hay que ser cuidadosos ya que en este proceso se corre el riesgo de dañar al embrión.

La inmersión en agua caliente fue efectiva para las semillas de Dodonaea viscosa recolectadas en la Mixteca Alta. El porcentaje de germinación reportado en este trabajo fue superior al obtenido por otros autores que usaron la misma técnica (González y Camacho, 1994; Phartyal et al., 2005). Esta diferencia podría deberse a variaciones entre poblaciones o entre años de producción (Gutterman, 1980), ya que en diferentes regiones del mundo Dodonaea viscosa presenta distintos requisitos para la germinación, lo que sugiere que hay una gran variación geográfica en los mecanismos que determinan su latencia. En algunos casos se ha encontrado que la escarificación mecánica es más eficiente que la sumersión en agua caliente (Phartyal et al., 2005), pero nosotros encontramos porcentajes tan altos de germinación que parece improbable que la apertura de la testa pueda mejorar nuestro resultado sustancialmente.

Para Arctostaphylos pungens y Juniperus flaccida se logró obtener una germinación de al menos $60 \%$, valor relativamente alto para estas especies (Young y Young, 1992; Cervantes et al., 2001). Arctostaphylos pungens no pertenece a una familia que incluya especies con una cubierta impermeable y Juniperus flaccida puede responder a tratamientos con giberelinas, lo que implica que su testa es permeable (Baskin y Baskin, 2000, 2004). En consecuencia, es de suponer que estas especies únicamente presentan latencia fisiológica, a pesar de que tienen una cubierta dura. Sin embargo, la escarificación en ácido quizá redujo la dificultad que el acto de perforar la testa representa para un embrión inmaduro. No obstante, la lenta germinación de ambas especies y su capacidad germinativa relativamente baja nos sugieren que la escarificación ácida fue insuficiente para compensar la latencia fisiológica en el total de la población de semillas usada (Baskin y Baskin, 2004). Nuestra metodología para hacerlas germinar no incluyó la estratificación o la aplicación de giberelinas exógenas que promovieran la maduración del embrión. Aun así, observamos que al contacto con el ácido se desprendió una gran cantidad de calor, lo que también pudo haber promovido la germinación, ya que se ha reportado que ambas especies germinan en el campo después de incendios donde se hayan alcanzado temperaturas altas (Hodgkinson, 1991).

La respuesta negativa a la escarificación mecánica en Quercus castanea sugiere que las semillas fueron dañadas por este tratamiento. En ocasiones, la escarificación puede permitir el paso acelerado de agua a la semilla y causar daño imbibicional (Bewley y Black, 1994). Por otra parte, se ha reportado que esta especie tiene latencia fisiológica acompañada de latencia morfológica, es decir, que los embriones no están completamente desarrollados (ZavalaChávez y García, 1996). Sin embargo, nosotros observamos que las semillas recién colectadas del árbol son 
capaces de germinar, ya que un lote de bellotas mojadas por la lluvia germinó en menos de una semana después de la colecta. Esto confirmó las propuestas de Gosling (1989) y Zavala-Chávez (2004) en el sentido de que las bellotas germinan mejor recién colectadas. Es posible que la deshidratación que las semillas pudieran haber sufrido entre la colecta y la siembra haya reducido su capacidad germinativa (Rodríguez et al., 2000).

Algunas semillas de Quercus deserticola son capaces de germinar aun cuando no se les aplique ningún tratamiento. Esto pudiera ser el resultado de que la especie no presenta ninguna forma de latencia, tal como lo reporta Zavala-Chávez (2004). Sin embargo, apenas se alcanzó un porcentaje de germinación de $50 \%$ a los 40 días de incubación. Esto puede sugerir la presencia alguna forma de latencia, pero no podemos afirmarlo con certeza ya que sólo se aplicó el tratamiento control en esta especie, y no sabemos si algún tratamiento pre-germinativo pudiera incrementar sus porcentajes de germinación. Otra posibilidad es que exista de latencia fisiológica distribuida diferencialmente en la población. Este tipo de latencia se rompe masivamente con algún tratamiento, o bien gradualmente después de un tiempo prolongado de incubación, por lo que la respuesta germinativa es lenta (Baskin y Baskin, 2004). Alternativamente, las especies de este subgénero pierden fácilmente su viabilidad cuando alcanzan bajos niveles de humedad, por lo que el periodo de un mes entre la colecta y la siembra pudo haber causado daño por deshidratación; este tipo de daños usualmente son reparados durante un periodo prolongado de incubación (Rodríguez et al., 2000).

La información generada en este estudio permite lograr la germinación exitosa de especies importantes para la restauración. Esto no implica que se trate de los mejores métodos posibles, ya que no se exploró en detalle todo el universo de tratamientos pre-germinativos potenciales. Sin embargo, se trata de técnicas de fácil aplicación y, en general, alta efectividad. En muchos casos se trata de datos básicos que faltan en la literatura, no sólo porque abordamos especies para las que no hay reportes previos, sino porque en algunos casos la información generada en un sitio no es aplicable en otro. Éste fue el caso de Dodonaea viscosa, especie cuyas semillas germinan aplicando una técnica diferente de la empleada en otras regiones, y Quercus castanea, para la cual se había reportado latencia fisiológica. El desarrollo de métodos apropiados para hacer germinar semillas de especies nativas es un requisito indispensable para lograr establecer un programa exitoso de restauración.

\section{Agradecimientos}

Armando Gutiérrez apoyó durante la colecta de las semillas empleadas. Itzel Baca Ibarra ayudó durante los experi- mentos. Agradecemos al Posgrado en Ciencias Biológicas con orientación a la restauración y al proyecto SEMARNATCONACyT (SEMARNAT-2002-C01-0591) por haber proporcionado recursos financieros para este estudio, y a la comunidad de Concepción Buenavista por su apoyo invaluable para la realización de este trabajo.

\section{Literatura citada}

Baskin J.M. y Baskin C.C. 1998. Seeds. Ecology, Biogeography, and Evolution of Dormancy and Germination. Academic Press, San Diego.

Baskin J.M. y Baskin C.C. 2004. A classification system for seed dormancy. Seeds Science Research 14:1-17.

Baskin J.M., Baskin C.C. y Li X. 2000. Taxonomy, anatomy and evolution of physical dormancy in seeds. Plant Species Biology 15:139-152.

Baskin J.M., Davis B.H., Baskin C.C., Gleason S.M. y Cordell S. 2004. Physical dormancy in seeds of Dodonaea viscosa (Sapindales, Sapindaceae) from Hawaii. Seeds Science Research 14:81-90.

Bewley J.D. y Black M. 1994. Seeds. Physiology of Development and Germination. 2a ed. Plenum Press, Nueva York.

Burrows C.J. 1995. Germination behavior of the seeds of six New Zealand woody plant species. New Zealand Journal of Botany 33:365-377.

Camacho M.F. y Bustillo O.H. 1988. Prospección del chapuixtle como arbusto topiario para el área urbana del Distrito Federal. Memorias de la Reunión Científica Forestal y Agropecuaria del Distrito Federal, CIFAP-DF, Instituto Nacional de Investigaciones Forestales y Agropecuarias, México, D.F.

Camacho M.F. y Morales V.G. 1992. Métodos para el análisis del efecto de tratamientos sobre la germinación. Memoria de la Reunión Científica Forestal y Agropecuaria del Campo Experimental. Publicación especial 1. SARH, Instituto Nacional de Investigaciones Forestales y Agropecuarias, CECOY, México, D.F.

Camargo-Ricalde S.L., Dhillion S.S. y García-García V. 2004. Phenology, and seed production and germination of seven endemic Mimosa species (Fabaceae-Mimosoideae) of the Tehuacán-Cuicatlán Valley, México. Journal of Arid Environments 58:423-437.

Carlson J.R. y Sharp W.C. 1975. Germination of high elevation manzanitas. Tree Planters' Notes 26:10-11.

Cervantes V. 1996. La reforestación de la montaña de Guerrero: una alternativa con leguminosas nativas. Tesis de Maestría, Facultad de Ciencias, Universidad Nacional Autónoma de México, México, D.F., 127 pp.

Cervantes V., Arriaga V., Meave J. y Carabias J. 1998. Growth analysis of nine multipurpose woody legumes native from southern Mexico. Forest Ecology and Management 110:329341.

Cervantes V., López-González M., Salas-Nava N. y HernándezCárdenas G. 2001. Técnicas para Propagar Especies Nativas de Selva Baja Caducifolia y Criterios para Establecer Áreas de Reforestación. Facultad de Ciencias, Universidad Nacional Autónoma de México, México, D.F.

Cervantes V., Salas-Nava N., López M. y Vázquez-Yanes C. 1998. 
Gilberto MartíneZ-Pérez, Alma Orozco-Segovia y Carlos Martorell

Propagación de leguminosas leñosas y herbáceas a partir de semillas. VII Congreso Latinoamericano de Botánica y XIV Congreso Mexicano de Botánica, México, D.F.

Crawley J.M. 1993. GLIM for Ecologists. Blackwell Scientific, Oxford.

Cruz-Cisneros R. y Rzedowski J. 1980.Vegetación de la cuenca del río Tepelmeme, Alta Mixteca, Estado de Oaxaca, (México). Anales de la Escuela Nacional de Ciencias Biológicas, Instituto Politécnico Nacional 22:19-84.

Djavanshir K. y Fechner G.H. 1975. Epicotyl and hypocotyl germination of eastern redcedar (Juniperus virginiana) and Rocky Mountain juniper (Juniperus scopulorum). Forest Science 22:261-266.

Everitt J.H. 1983. Seed germination characteristics of two woody legumes (retama and twisted acacia) from South Texas. Journal of Range Management 36:411-414.

Flores J. y Jurado E. 1998. Germination and early growth traits of 14 plants species native to northern Mexico. Southwestern Naturalist 43:40-46.

González-Castañeda J., Angoa-Pérez M.V., Frías-Hernández J.T., Olalde-Portugal V., Flores-Ancira E., Terrones-Rincon T.R.L., Van Cleemput O. y Dendooven L. 2004. Germination of seeds of huisache (Acacia schaffneri) and catclaw (Mimosa monancistra) as affected by sulphuric acid and mechanical scarification and subsequent growth and survival in a greenhouse and field experiment. Seed Science and Technology 32:727-738.

González K.V. y Camacho M.F. 1994. Avances en la propagación de cuatro especies presentes en El Pedregal de San Ángel D.F. En: Rojo A. Ed. Reserva Ecológica del Pedregal de San Ángel. Ecología, Historia Natural y Manejo, pp. 403-410, Universidad Nacional Autónoma de México, México, D.F.

González-Zertuche H.M.L. y Orozco-Segovia A. 1996. Métodos de análisis de datos de la germinación de semillas, un ejemplo: Manfreda brachystachya. Boletín de la Sociedad Botánica de México 58:37-52.

Gosling J.M. 1989. The effect of drying Quercus robur acorns to different moisture contents. followed by storage either with or without imbibition. Forestry 62:124-136.

Gutterman Y. 1980. Influences on seed germinability: phenotypic maternal effects during seed maturation. Israel Journal of Botany 29:10-117.

Hodgkinson K.C. 1991. Shrub recruitment response to intensity and season of fire in a semi-arid woodland. Journal of Applied Ecology 28:60-70.

Hodgkinson K.C. y Oxley R.E. 1990. Influence of fire and edaphic factors on germination of the arid zone shrubs. Australian Journal of Botany 38:269-279.

Hussain F., Shaukat S., Ilahi I. y Zakeriva M.Q. 1991. Note on the germination behaviour of Dodonaea viscosa (Lem) Jaq. Science Khyber 4:45-49.

Jurado E., Aguirre O., Flores J., Navar J., Villalón H. y Wester D. 2000. Germination in Tamaulipan thornscrub of northeastern Mexico. Journal of Arid Environments 46:413-424.

Keeley J.E. 1987. Role of fire in seed germination of woody taxa in California Chaparral. Ecology 68:434-443.

Martorell C. 1995. Consecuencias ecológicas y alternativas del uso de la leña en la comunidad de Los Reyes Metzontla. Tesis de Licenciatura (Biología), Facultad de Ciencias, Universidad Nacional Autónoma de México, México, D.F., 136 pp.

Marroquín J.S. 2001. Sapindaceae. En: Rzedowski J. y
Rzedowski G. Eds. Flora Fanerogámica del Valle de México, Vol. II, pp. 381-385, Escuela Nacional de Ciencias Biológicas e Instituto de Ecología, México, D.F.

Martínez-Pérez G. 2004. Efecto de la perturbación crónica sobre la integridad biológica de las comunidades vegetales de Concepción Buenavista, Oaxaca. Tesis de Licenciatura (Biología), Facultad de Ciencias, Universidad Nacional Autónoma de México, México, D.F., 74 pp.

Medina-Lemus R. y Dávila-Aranda P. 1997. Flora del Valle Tehuacán-Cuicatlán. Gymnospermae Lindl. Fascículo 12. Instituto de Biología, Universidad Nacional Autónoma de México, México, D.F.

Murguía-Sánchez G. 1986. Estudio comparativo de semillas maduras de dos especies arbóreas del género Ipomoea (Convolvulaceae). Tesis de Licenciatura (Biología), Facultad de Ciencias, Universidad Nacional Autónoma de México, México, D.F., 61 pp.

Niembro-Rocas A. 1986. Árboles y Arbustos Útiles de México. Limusa, México, D.F.

Odion D. y Tyler C. 2002. Are long fire-free periods needed to maintain the endangered, fire recruiting scrub Arctostaphylos morroensis (Ericaceae)? Conservation Ecology 62:395-408.

Orozco-Almanza M.S., de Leon-García L.P., Grether R. y GarcíaMoya E. 2003. Germination of four species of the genus Mimosa (Leguminosae) in a semi-arid zone of Central Mexico. Journal of Arid Environments 55:75-92.

Pardos J.A. y Lázaro G. 1983. Germination aspects of Juniperus oxycedrus. Anales del Instituto Nacional de Agricultura Madrid 7:153-163.

Phartyal S.S., Baskin J.M., Baskin C.C. y Thapliyal R.C. 2005. Physical dormancy in seeds of Dodonaea viscosa (Sapindaceae) from India. Seed Science and Research 15:5961.

Pugnaire F., Hasse P., Puigdefábregas M., Cueto S., Clark C. y Incoll L. 1996. Facilitation and succesion under the canopy of leguminous scrub, Retama sphaerocarpa, in a semi-arid environment in south-east Spain. Oikos 76:455-464.

Qadir S.A. y Lodhi N. 1971. Germination behavior of seeds of some common shrubs. Journal of Science 1:84-97.

Rey-Cruz O. 1985. Influencia de los glucósidos de Turbina corymbosus en la germinación de semillas de Ipomoea murucoides. Tesis de Licenciatura (Biología), Facultad de Ciencias, Universidad Nacional Autónoma de México, México, D.F., 74 pp.

Rico-Arce L. y Rodríguez A. 1998. Flora del Valle TehuacánCuicatlán. Mimosaceae R. Br. Tribu Acacieae Benth. Fascículo 20. Instituto de Biología, Universidad Nacional Autónoma de México, México, D.F.

Rodríguez R., Centeno M.L., Cañal M.J., Rodríguez A., Fernández B. y Fraga M.F. 2000. Physiological basis of plant ageing. Problems and solutions for micropropagation of gymnosperms and angiosperms selected mature trees. En: Espinel S. y Ritter E. Eds. Applications of Biotechnology to Forest Genetics, pp. 411-424, Diputación Foral de Álava, Vitoria.

Rolston M.P. 1987. Water impermeable seed dormancy. The Botanical Review 44:356-436.

Rosa S.G. y Ferreira A.G. 2001. Germinação de sementes de plantas medicinais lenhosas. Acta Botanica Brasilica 15:147154.

SEMARNAT [Secretaría del Medio Ambiente y Recursos 
Naturales]. 2000. Disponible en: <www.semarnat.gob.mx/pfnm> Vázquez-Villagrán M.L. 2000. Flora del Valle TehuacánCuicatlán. Fagaceae Dumort. Fascículo 28. Instituto de Biología, Universidad Nacional Autónoma de México, México, D.F.

Vázquez-Yanes C., Batis-Muñoz A.I., Alcocer-M. I., Gual-Díaz M., y Sánchez-Dirzo C.1995. Árboles y Arbustos Nativos Potencialmente Valiosos para la Restauración Ecológica y la Reforestación. CONABIO, México, D.F.

Vázquez-Yanes C. y Batis-Muñoz A.I. 1996. Adopción de especies nativas valiosas para la restauración y reforestación. Boletín de la Sociedad Botánica de México 58:75-84.

Vázquez-Yanes C. y Cervantes V. 1993. Estrategias para la reforestación con árboles nativos de México. Ciencia y Desarrollo 19:52-58.

Virginia R.A. y Jarrel W.M. 1983. Soil properties in a mesquite- dominated Sonora desert ecosystem. Soil Science Society of America Journal 47:138-144.

Vora R. 1989. Seed germination characteristics of selected native plants of the lower Rio Grande Valley, Texas. Journal of Range Management 42:36-40.

Young J.A., Evans J.D., Budy J.D. y Palmquist D.E. 1988. Stratification of seeds of Western and Utah juniper. Forest Science 34:1058-1060.

Young J.A. y Young C.G. 1992. Seeds of Woody Plants in North America. Dioscorides Press, Portland.

Zavala-Chávez F. 2004. Desecación de bellotas y su relación con su viabilidad y germinación de nueve especies de encinos mexicanos. Ciencia Ergo Sum UAEM 11:177-185.

Zavala-Chávez F. y García E. 1996. Frutas y Semillas de Encinos. Universidad Autónoma de Chapingo, Texcoco.

Fecha de recepción: 28 de febrero de 2006

Versión corregida: 10 de octubre de 2006

Aceptado: 11 de octubre de 2006 
Apéndice 1. Obtención de las curvas de germinación.

Para garantizar la independencia de los datos requerida por la mayoría de las pruebas estadísticas empleamos la probabilidad de que una semilla que no ha germinado lo haga en el tiempo $t$, esto es, la probabilidad condicional

$$
\operatorname{Pr}\left(G_{t} \mid N_{t-1}\right)
$$

Donde $G_{t}$ es el evento "germinar en el tiempo $t^{\prime}$ y $N_{t-1}$ es el evento "no haber germinado hasta el tiempo t-1". Estas probabilidades se generaron a través de una regresión logística que reconoce dos atributos de las proporciones: (1) que su error sigue una distribución binomial, y (2) que están acotadas entre 0 y 1, lo que se logra empleando una transformación logística de los datos (Crawley, 1993). El correcto funcionamiento del método requiere que los datos sean tomados en intervalos regulares de tiempo.

Para conocer la probabilidad de germinar al tiempo $t$, , que es un parámetro fácilmente interpretable desde el punto de vista biológico, además de ser empleado en la mayoría de los estudios de germinación, podemos aplicar el teorema de Bayes

$$
\operatorname{Pr}\left(G_{t} \mid N_{t-1}\right)=\frac{\operatorname{Pr}\left(N_{t-1} \mid G_{t}\right) \operatorname{Pr}\left(G_{t}\right)}{\operatorname{Pr}\left(N_{t-1}\right)}
$$

Puesto que una semilla no puede germinar dos veces, es seguro que semilla que germine al tiempo $t$ no ha germinado con anterioridad, es decir

$$
\operatorname{Pr}\left(N_{t-1} \mid G_{t}\right)=1
$$

Por lo tanto, sustituyendo (2) en (1) y despejando

$$
\operatorname{Pr}\left(G_{t}\right)=\operatorname{Pr}\left(G_{t} \mid N_{t-1}\right) \operatorname{Pr}\left(N_{t-1}\right)
$$

Como las semillas sólo germinan una vez, podemos asegurar que $G_{i}$ y $G_{j}$ son eventos excluyentes, por lo que sus probabilidades son aditivas. Por lo tanto, la probabilidad de no haber germinado en el tiempo $t$ - 1 puede calcularse como:

$$
\operatorname{Pr}\left(N_{t-1}\right)=1-\sum_{i=1}^{t-1} \operatorname{Pr}\left(G_{i}\right)
$$

Para estimar las probabilidades de germinación podemos sustituir la ecuación (4) en la (3) para generar la fórmula recursiva:

$$
\operatorname{Pr}\left(G_{i}\right)=\operatorname{Pr}\left(G_{t} \mid N_{t-1}\right)\left(1-\sum_{i=1}^{t-1} \operatorname{Pr}\left(G_{i}\right)\right)
$$

Finalmente, para calcular el primer elemento de la serie recursiva consideramos que al inicio del experimento ninguna semiIla ha germinado, es decir

$$
\operatorname{Pr}\left(N_{0}\right)=1
$$

por lo que, sustituyendo en (5),

$$
\operatorname{Pr}\left(G_{1}\right)=\operatorname{Pr}\left(G_{1} \mid N_{0}\right)
$$

dato que se obtiene directamente de la regresión. 